\title{
Construcción de Conocimiento Mapuche y su relación con el Conocimiento Escolar*
}

\author{
Construction of Mapuche knowledge and its relation with school knowledge
}

Construção do conhecimento mapuche e sua relação com o conhecimento escolar

\author{
Segundo Quintriqueo $M .^{\mathrm{a}}$ y Héctor Torres $C$. $^{\mathrm{a}}$ \\ ${ }^{a}$ Facultad de Educación, Universidad Católica de Temuco. Casilla 15 D, Temuco. Correo electrónico: \\ squintri@uct.cl
}

\begin{abstract}
RESUMEN
El artículo tiene como objetivo analizar la construcción de conocimiento mapuche y su relación con el conocimiento escolar disciplinario en los procesos educativos interculturales. El supuesto es que existen lógicas de pensamientos presentes en los procesos culturales de construcción de conocimientos y los sistemas de saberes, tanto occidental como mapuche. Estos son deseables de considerar en la implementación de la educación intercultural, en el ámbito de la enseñanza de las ciencias en escuelas situadas en comunidades mapuches de la IX Región de La Araucanía. Los resultados muestran una descripción sobre las lógicas de construcción de conocimientos tanto mapuche como occidental, tomando como base el discurso de kimches (sabios), para la contextualización de la enseñanza-aprendizaje en procesos de implementación de la educación intercultural en contexto mapuche.
\end{abstract}

Palabras clave: lógicas del conocimiento, sistema de saberes, educación intercultural.

\begin{abstract}
The aim of this article is to analyze the construction of Mapuche knowledge and its relationship with the disciplinary knowledge of schools within the intercultural processes of education. The premise is that there are certain logics of thought under the cultural processes of construction and systems of knowledge, both in western and Mapuche contexts. The previous factors are worth considering in the implementation of intercultural education, in the particular contexts of science teaching in schools located in Mapuche communities of the IX region of La Araucania. The results provide a description of this logic of construction of knowledge in both western and Mapuche realities; considering as a building block the speeches of Kimches (wise men) for the teaching-learning contextualization in processes of intercultural education implementation within a Mapuche context.
\end{abstract}

Key words: logic of knowledge, epistemology, intercultural education.

\section{RESUMO}

Objetiva-se analisar a construção do conhecimento mapuche e sua relação com o conhecimento escolar oferecidos pelas disciplinas nos processos educativos interculturais. O pressuposto é que existem lógicas de pensamentos presentes nos processos culturais de construção de conhecimentos e sistemas de saberes tanto ocidental quanto mapuche. Estes são desejáveis na implementação da educação intercultural, no campo do ensino de Ciências em escolas de comunidades mapuches da IX Região de La Araucanía. Resultados mostram uma descrição da lógica de construção de conhecimentos tanto mapuche como ocidental baseando-se no discurso de kimches (sábio) para a contextualização do processo ensino-aprendizagem nos andamentos da implementação da educação intercultural em contexto mapuche.

Palavras chave: lógica do conhecimento, sistema de saberes. educação intercultural.

* El artículo es financiado por el Fondo Nacional de Desarrollo Científico y Tecnológico (FONDECYT), proyecto REGULAR Nº 1110489 . 


\section{INTRODUCCIÓN}

Este artículo tiene por objeto analizar la distancia que presentan los modos de construcción de conocimientos mapuches y los conocimientos disciplinarios en ciencias, teniendo como fundamento el discurso de educadores/as tradicionales denominados kimches, ${ }^{1}$ que podrían aportar a la generación de una propuesta educativa contextualizada en escuelas de Educación Básica de la IX región de La Araucanía. Los conocimientos en la enseñanza de las ciencias corresponden a los sectores Ciencias Naturales e Historia, Geografía y Ciencias Sociales, reconocidos como oficiales para la cultura global, negando aspectos de otras culturas en el currículum escolar.

En la investigación se plantea el supuesto de que los kimches de las comunidades que participan en la implementación de la educación intercultural bilingüe, conocen los contenidos y finalidades educativas vigentes en las comunidades mapuches para la formación de las personas. Estos sistemas de saberes poseen su propia lógica, la que les permite conocer y comprender la realidad en sus diferentes dimensiones: naturales, sobrenaturales y espirituales, las que configuran una unidad indivisible y están en la base del conocimiento educativo mapuche.

Sin embargo, históricamente la escolarización de niños mapuches en el contexto de las escuelas del sistema educativo escolar, se realiza en base a contenidos y finalidades educativas occidentales. Así, cuando los niños se incorporan a la educación escolar, adquieren un conjunto de elementos culturales que no les pertenecen y que pasan a formar parte de las dimensiones ocultas del currículo (oculto, negado u omitido). Además, los conocimientos se organizan y transmiten con códigos que tampoco les pertenecen. En efecto, son conocimientos que se estructuran en base a la racionalidad epistémica occidental. La coexistencia de hecho de estas lógicas para conocer, explicar y comprender la realidad es inevitable y se nos presenta como un obstáculo epistemológico, necesario de explicitar para desarrollar un currículum contextualizado interculturalmente. Ambas categorías y racionalidades de conocimientos, constituyen aquí objeto de reflexión para la generación de conocimientos que fundamenten la educación intercultural.

\section{MARCO DE REFERENCIA}

La distancia epistemológica entre los saberes culturales mapuches y el conocimiento escolar en el sector de Ciencias se sustentan en dos modos de construcción (Quintriqueo y Cárdenas, 2010). Por una parte, el conocimiento cultural mapuche se construye en una relación directa del sujeto con el medio natural, social, cultural y espiritual, en las dimensiones subjetiva e intersubjetiva para comprender la realidad. Por otra parte, los conocimientos escolares son una síntesis del conocimiento científico, considerados universalmente válidos, que prioriza lo occidental como lo único, lo moderno y lo verdadero, para comprender la realidad.

Los kimches en su calidad de sabios son portadores de saberes y conocimientos fundados en la memoria individual y social mapuche, quienes son incorporados con el nombre de Educadores Tradicionales a la escuela; desde el Ministerio de Educación para apoyar a profesores de ascendencia mapuche y no mapuche en la implementación de la educación intercultural bilingüe. 
De esta forma, "las culturas autóctonas se definen como míticas y son algo que se debe superar por ser un obstáculo para el desarrollo universal” (Restrepo, 2004: 58). En efecto, la distancia epistemológica observada entre el conocimiento escolar y el conocimiento mapuche, se relaciona con las dificultades que tienen los alumnos para asimilar un marco interpretativo científico para el análisis de la realidad. Es posible que esto se deba a la desvalorización de los saberes mapuches en el medio escolar, por ser considerados una fuente contaminadora y obstaculizadora para el aprendizaje y la enseñanza del conocimiento escolar (Rodrigo y Arnay, 1997; De Camilloni, 2001; Guerra, 2006; Schmelkes, 2006; Sagastizábal, 2006).

Es bajo este marco que se considera necesario abordar el concepto de epistemología, el que proviene del griego Episteme -conocimiento- y logos -tratados-. A la base de esta primera aproximación se asume que la epistemología es el estudio crítico del conocimiento, de sus fundamentos, de sus principios, de sus métodos, de sus resultados y la manera de establecer su validez (Legendre, 1993). Disciplina que tiene como objeto de estudio el conocimiento científico, cuyos aspectos más particulares los constituyen los problemas del conocer, del saber y los conceptos relacionados con el sentido y la inteligencia, para comprender la realidad contextual (Nadeau, 2009). En ese contexto, las palabras no tienen realidad en sí mismas y se les atribuye una valoración respecto de su pertenencia a la memoria individual y social, entendiéndose como creaciones sociales. Según Ardoino (2005), actualmente es posible encontrar epistemologías diversas, concurrentes, contradictorias y diferentes. Por tanto, el sentido de las palabras, transmiten epistemologías distintas.

Desde el punto de vista del conocimiento mapuche, la epistemología la definimos como konünpa kimün, donde: 1) kon: indica la acción de entrar, lograr un conocimiento nuevo o traer aquello que está en la memoria individual y social; 2) ün: terminación verbal en primera persona, en tiempo pasado; y 3) pa: partícula que asevera la realización de la acción del verbo que le precede. Por su parte: a) kim: refiere a aquello que es sabido, información, saber y conocimiento; y 2) mün: refiere al objeto del saber y del conocimiento (Quilaqueo, Fernández y Quintriqueo, 2010). Entonces, desde la lógica mapuche, el conocimiento se relaciona con el sentido de las palabras, a las que se le atribuye una valoración para analizar los discursos asociados a la memoria individual y social que definen la racionalidad del pensamiento y forma de organizar el saber como una creación social sobre el estudio de la posibilidad y origen del conocimiento propio y la manera de establecer su legitimación para comprender la realidad (Quintriqueo y Cárdenas, 2010). Entonces, los problemas centrales de una epistemología para una educación intercultural en contexto mapuche es develar los tipos de conocimientos educativos propios, los criterios de verdad, las fuentes de orígenes y, en definitiva, los diferentes elementos involucrados en la relación entre el sujeto que conoce y el objeto de conocimiento. En efecto, el conocimiento se ubica como una dimensión humana, considerada como un repertorio de experiencias culturales, acumuladas en la memoria individual y colectiva a través del tiempo, y expresada en los discursos orales o escritos, según sea el tipo de cultura.

En este proceso, no se excluyen los aspectos metacognitivos ni las capacidades de reflexionar acerca del propio conocimiento o acerca de las realidades objetivas o subjetivas, en cualquiera de sus dimensiones (Izquierdo, 1999). Desde esta perspectiva, Hessen (2003) destaca que en toda relación de conocimiento es posible identificar los siguientes elementos esenciales: 
1) Todo acto de conocimiento se realiza en una relación sujeto-objeto (S-O). Dentro de esta concepción, esta dualidad se acepta como un elemento necesario del conocimiento (Dualismo epistemológico).

2) Del mismo modo, la relación entre Sujeto-Objeto se presenta de manera reversible, transformándose en correlación. Es decir, algo es objeto cognoscible, sólo para un sujeto cognoscente y a la inversa. En esta correlación la función del sujeto es aprehender las cualidades del objeto. Para ello, el sujeto debe trascender del polo de su 'subjetividad' hacia el objeto, el que a su vez trasciende hacia el sujeto.

3) En esta trascendencia se forma una imagen del objeto en el sujeto o una representación abstracta del objeto en el sujeto $(\mathrm{O}-\mathrm{S})$, que contiene las principales propiedades de él. Desde esta perspectiva el conocimiento no es nada más que una transferencia de las propiedades del objeto hacia el sujeto.

En las culturas originarias, tales como la mapuche, la dualidad S-O, desde el punto de vista epistemológico y ontológico, está constituida por elementos coherentes, representados en la correlación de pares de realidades multidimensionales. Por ejemplo, unidades tales como hombre-mujer, hombre-naturaleza, hombre-mujer-comunidad, hombre-mujer-comunidad-fuerzas naturales, seres sobre naturales y espirituales, van configurando realidades con caracteres hologramáticos (Izquierdo, 1999), que se van constituyendo en sistemas complejos. Debe entenderse que este carácter unitario de las culturas originarias, no se refiere a los dualismos epistemológicos ni ontológicos propios de la cultura occidental. En las culturas originarias es más propio hablar de un monismo epistemológico y ontológico, en cuya racionalidad el conocimiento se construye como un sistema de saberes subjetivo, intersubjetivo e interrelacionado.

La concepción cíclica del espacio-temporalidad, según el conocimiento mapuche, está organizada en base a principios y finalidades coherentemente definidos. Esto obliga a la búsqueda de otros referentes teóricos y paradigmas para responder a los problemas de construcción de saberes y conocimientos indígenas. Desde esta perspectiva, cualquier análisis del conocimiento construido por los pueblos indígenas debiera realizarse a partir de sus particularidades. El problema que nos plantean esta postura es que la sociedad occidental ha construido su historia en forma lineal, monocrónica, la que se ha universalizado y postulado como única y verdadera (Quintriqueo y Cárdenas, 2010). En la concepción occidental se reconoce el pasado como algo que quedó atrás, ya superado; el futuro como algo venidero, desconocido e incierto; y el presente como algo inexistente. Esta concepción transversaliza los diferentes aspectos de la cultura occidental de la cual la escuela no está exenta. En cambio, en el caso de los pueblos originarios y en este caso la comunidad mapuche, se rigen por una noción témporo-espacial no lineal, sino más bien cíclica, que considera la dinámica del orden natural del universo con sus procesos de contracción y expansión (Quintriqueo y Cárdenas, 2010). En esta lógica, la dimensión temporal del pasado se ubica delante de nosotros donde lo sabido, lo conocido, señala el camino que se va a seguir. El futuro está detrás como algo que aún no viene, aparecerá detrás del otro y de nosotros como algo desconocido, discutible y cuestionable. El presente constituye la convergencia entre lo discutible y lo cuestionable, lo que resultará como un sistema de saber que permite comprender la realidad (Restrepo, 2004, Quintriqueo y Cárdenas, 2010; Torres y Quilaqueo, 2010). 
En el proceso de construcción de conocimiento mapuche, en la relación directa con la naturaleza, es central la dimensión espiritual de la dualidad objeto-sujeto. Esta relación es expresada en el sentido y significado totalizante pero situacional de la práctica sociocultural, caracterizada por una contextualización permanente de los contenidos, nociones y conceptos para comprender la racionalidad propia (Quintriqueo, 2010). La finalidad es la articulación permanente de la relación del hombre con la naturaleza para definir un contexto donde desenvolverse y el texto o el contenido construido es el sentido de las palabras en el discurso de los kimches (sabios). Los elementos esenciales que los kimches definen en sus ideas son los criterios y los principios centrales en una relación totalizante hombre-naturaleza-comunidad-cosmos, donde el hombre es un componente más del mundo. En esta perspectiva, el diálogo intercultural supone una contextualización de ambas concepciones epistemológicas en base a los contenidos y las finalidades educativas, para la formación de personas en el medio escolar y familiar.

\section{METODOLOGÍA}

La investigación es de carácter descriptiva (Rodríguez, Gil y García, 1999) y se enmarca en la Metodología de la Investigación Educativa (Gauthier, Desbiens, Malo, Martineau y Simard, 1997; Bisquerra, 2004). Su utilización se justifica dado que la pregunta central se vincula directamente con la educación en la relación entre el conocimiento mapuche y el conocimiento occidental en el marco del currículum escolar. La comprensión de ambas lógicas de conocimientos tiene por objeto dilucidar el modo de construcción e incorporación de conocimientos educativos mapuches a la educación escolar, desde un enfoque educativo intercultural.

Los sujetos considerados en la muestra corresponden a: ocho kimches (cuatro hombres y cuatro mujeres) de Padre Las Casas y 24 kimches (12 mujeres y 12 hombres) de la comuna de Chol-Chol. En total suman 32 kimches, quienes respondieron individualmente entrevistas semiestructuradas y semidirigidas (Bisquerra, 2000). Se utilizó una técnica de muestreo intencionado, mediante el uso de un Diseño de Casos Múltiples, seleccionados como Casos Típicos, basados en los criterios de edad, pertenencia étnica, género y áreas geográficas. Las razones teóricas y metodológicas para implementar esta modalidad fueron:1) Justificar que el saber y el conocimiento educativo mapuche están validados por la memoria social de la familia mapuche, en particular por los kimches; 2) Confirmar que los kimches permiten dar una mejor lectura sobre la presencia y ausencia de contenidos educativos mapuches en el medio escolar; 3) Identificar y describir los contenidos que subyacen en la relación de parentesco como elementos de la identidad sociocultural mapuche; y 4) Identificar el punto de vista de miembros de la comunidad mapuche, con respecto a la distancia epistemológica entre el conocimiento mapuche y el occidental (disciplinar) en la formación de niños y jóvenes de ascendencia mapuche en la educación escolar (Quintriqueo, 2002).

Los principales contenidos que se abordaron en las entrevistas, fueron los saberes y conocimientos mapuches y su relación con los conocimientos escolares en el contexto de escuelas situadas en comunidades. Cada entrevista tuvo una duración aproximada de 30 a 45 minutos. 
Las informaciones fueron registradas mediante grabaciones de audio en la comunidad y observaciones etnográficas en el medio escolar. La transliteración de la información resultante se realizó en castellano. En este proceso, se escribió la información bajo la misma lógica en que los kimches la expresaron en sus discursos, en relación con los temas, categorías y dimensiones consignadas en la investigación. Los códigos expresan el significado de una categoría en el plan de análisis y se relacionan con las unidades temáticas, en cuyo proceso el entrevistado se representa con la letra ' $\mathrm{K}$ ', más el número que le corresponda, por ejemplo, K1 hace referencia a la entrevista del sujeto número uno. El ordenamiento de la información se realizó en base a las categorías de análisis explicitadas en el modelo de análisis. Es decir, es un reagrupamiento de datos y contenidos que se cuestionan y se analizan para develar sus significados en virtud de los propósitos de la presente investigación.

El análisis de la información (entrevistas, discursos), se realizó mediante el análisis de contenido (Krippendorff, 1990; Quivy y Campenhoudt, 1998) para develar núcleos de saberes centrales de orden abstracto, dotados de sentido y significado desde la perspectiva de los productores del discurso (Pérez 2000). Este procedimiento se implementa combinando el método clásico de análisis de contenido (Krippendorff, 1990) y los procedimientos de descripción de los mismos, según los códigos en el programa informático Atlas. ti 5.0..

\section{RESULTADOS}

Los resultados se relacionan con la categoría construcción de conocimientos culturales mapuches asociado a tres subcategorías: actividades de aprendizaje, ceremonias socioculturales y objetos de conocimiento.

\subsection{CATEGORÍA CONSTRUCCIÓN DE CONOCIMIENTOS CULTURALES MAPUCHES}

En la red conceptual de la Figura 1, se presenta la categoría construcción de conocimiento, asociada a elementos sociales y culturales presentes, según la lógica mapuche.

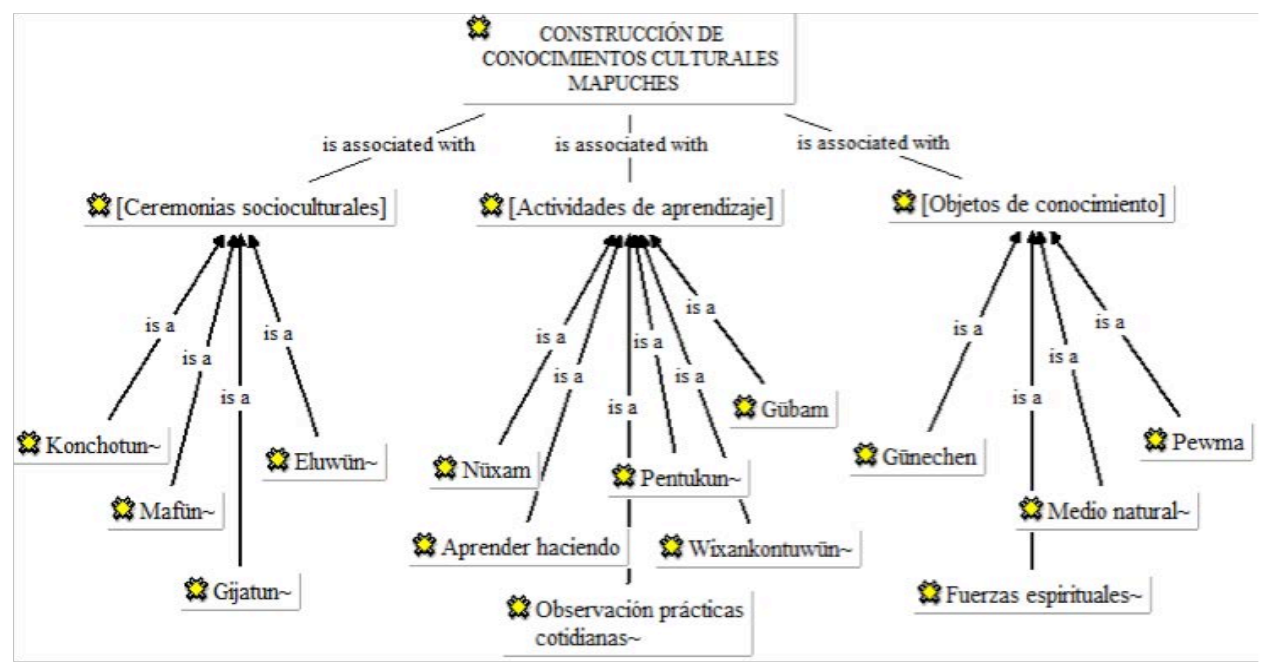

Figura 1. Categoría Construcción de conocimientos culturales mapuches 


\subsubsection{Subcategoría Actividades de aprendizaje}

La subcategoría Actividades de aprendizaje corresponde a los procesos relacionados con el aprendizaje de saberes y conocimientos que forman parte de la memoria individual y social de la familia mapuche. Es así que los kimches señalan las siguientes actividades de aprendizaje: el gübam (consejo a niños y jóvenes), el aprender haciendo, el wixakontuwün (visitarse), el pentukun (saludo protocolar) y la observación de prácticas cotidianas.

\begin{tabular}{|c|c|c|}
\hline Código & Frecuencia & \% \\
\hline Gübam & 21 & 22,8 \\
\hline Aprender haciendo & 19 & 20,7 \\
\hline Wixankontuwün & 17 & 18,5 \\
\hline Pentukun & 14 & 15,2 \\
\hline Nüxam & 11 & 11,9 \\
\hline Observación prácticas cotidianas & 10 & 10,9 \\
\hline Total & 92 & 100 \\
\hline
\end{tabular}

Tabla 1. Subcategoría Actividades de aprendizaje

El gübam (consejo a niños y jóvenes) es considerado una actividad que tiene como propósito formar a las personas. Es así que la frecuencia de uso obtenida por el código es de un $22,8 \%$. De esta manera, en el medio familiar mapuche los kimches (sabios) señalan que el gübam se realiza para que “...los hijos e hijas aprendan sobre las formas de pensamiento, lo que es un buen pensamiento, de respetar a sus familiares, a los mayores, a los padres, a los hermanos, a las hermanas" (K7 [15:15]). Entonces, el consejo permite enseñar a los niños, los valores, el respeto y la forma de pensar que es propia de la familia mapuche. El consejo adquiere una connotación destacada, siendo una acción continua en el tiempo y necesario para el crecimiento de las personas: "Todos los hijos no crecen iguales, pero desde el conocimiento mapuche, al hijo se le aconseja diariamente, para que logren mentalizar los valores y conocimientos" (K1 [45:45]).

El aprender haciendo se relaciona con actividades prácticas que realizan niños y jóvenes, con tal de aprender a desarrollar diferentes oficios en la familia y en los trabajos que forman parte del diario vivir, tanto al interior como exterior del hogar. Es así que la frecuencia de uso obtenida por el código es de un 20,7\%. De esta manera, un testimonio señala que, cuando iban a trabajar al campo, el padre le decía "tú vas a desparramar el trigo y yo voy a arar. Es decir, el padre quiso que su hijo practicase inmediatamente el trabajo del campo, pero guiado por él mismo" (K6 [30:30]). Mientras que en actividades desarrolladas por la mujer, se señala que “...cuando éramos niñas un poco más grande, practicábamos y nos gustaba mucho el trabajo en telar, para ello practicamos para aprender hacer el wachi (sistema entramado para tomar los hilos) y colocar el züwewe (palillos para separar los hilos)" (K11 [29:29]). Así, el aprender haciendo se aplica en diferentes situaciones de la vida familiar, donde los niños aprenden a cumplir diferentes oficios y responsabilidades. Por ejemplo, hacer un pentukun (saludo protocolar), una rogativa, el trabajo de la siembra y tejer en el telar. 
El Wixankontuwün (visitarse) es una actividad que permite a las personas compartir y reunirse como grupo familiar, código que obtiene una frecuencia de uso de un 18,5\%. La realización de esta actividad permite a las personas mapuches “...conocer el estado de salud, y el estado anímico de las personas. También cuando hay interés por tener una conversación" (K12 [27:27]). A través de las visitas, se mantiene la comunicación con familiares o personas cercanas a la familia, para así saber sobre su estado de salud o ánimo. Además, se fortalecen los lazos familiares señalándose que "son familias quienes se visitan, sean hermanos, o las tías y tíos paternos y maternos" (K10 [28:28]). El visitarse implica sentimientos de afectividad y cariño entre la familia, los que son aprendidos por los niños al relacionarse con otros miembros del grupo familiar.

El pentukun (saludo protocolar), corresponde a una conversación que se realiza entre dos personas, que permite dialogar sobre la familia, código que obtiene una frecuencia de uso de un $15,2 \%$. De esta forma, el pentukun implica según el testimonio de los kimches que "este tipo de conversación, es una forma de conocer a la otra persona $y$ de aprender a guardar información, hacer trabajar la memoria" (K12 [11:11]). El pentukun se utiliza para ofrecer un saludo formal a los familiares y no familiares. El propósito es conocer sobre los parientes, principalmente sobre la procedencia territorial y parental. Su realización implica una acción de conocer al otro. Es así que el pentukun se puede iniciar con una pregunta referida al lugar de origen y la procedencia sanguínea de la persona, de la familia. Cuál es el lugar de procedencia del abuelo paterno, de la abuela paterna, la abuela materna. En el proceso, quienes realizan el pentukun y quienes lo escuchan, están atentos a las respuestas para incorporar la información a la memoria. Los testimonios señalan que la idea es no olvidar la información, sino más bien guardarla: "Posteriormente, la persona que está con su papá, debiera preguntar de la misma forma, lugar de procedencia, de qué familia descendemos" (K12 [9:9]). A su vez, un aspecto relevante del pentukun es el valor asignado a la familia mapuche, asociado a los saberes e ideas que configuran la relación entre los parientes y su conocimiento sobre la procedencia territorial y su historia como comunidad. Las personas que realizan el pentukun y quienes participan de él desarrollan condiciones que definen el contexto, los temas de aprendizaje y disposiciones personales para el aprendizaje como lo señala un testimonio: "conversaban harto tiempo antes de hablar el tema central y yo me quedaba escuchándolos y era lindo" (K7 [18:18]).

El nüxam (conversación), corresponde a una actividad de aprendizaje en la que se enseña saberes y conocimientos a los niños y jóvenes, alcanzando el código un 11,9\% de las frecuencias. Para el desarrollo de la conversación, los kimches señalan que “...los niños se encuentran agrupados con los abuelos, quienes les conversan sobre cómo se alcanza el buen pensamiento mapuche..." (K1 [16:16]). De esta forma, por medio de la conversación los niños logran aprender diferentes contenidos educativos en el contexto familiar. Entonces, una disposición fundamental para que el niño aprenda es escuchar a la madre o al padre. En este proceso, los enseñantes en la familia mapuche conversan sobre un tema central, lo analizan y discuten para alcanzar un consenso sobre aspectos esenciales del contenido enseñado. Por ejemplo, un testimonio señala: "Yo, le digo a mis hijos que el foye (canelo) sirve para tal enfermedad, es un gran remedio, así les digo" (K4 [26:27]). Por lo tanto, en los niños y jóvenes se desarrolla una actitud de escuchar atentamente lo que dicen sus padres o abuelos, con tal de comprender los conocimientos que les son enseñados, tanto en el interior del hogar como en espacios comunitarios. 
La observación de prácticas cotidianas permite a niños y jóvenes participar de actividades que les permitan estar concentrados respecto a la acción que se realiza, para internalizar lo que observan, una frecuencia de uso del 10,9\%. Los kimches señalan que "los jóvenes deben observar y estar pendiente de lo que los mayores hacían o decían, ya que esa era la única forma adecuada de aprender estos temas" (K11 [14:15]). Este es un proceso de observación directa que involucra a los niños y jóvenes en el aprendizaje y, en efecto, las experiencias de relación con el medio natural, social, cultural y espiritual están en la base de la forma de concebir el conocimiento.

\subsubsection{Subcategoría Ceremonias socioculturales}

La subcategoría Ceremonias socioculturales se refiere a los procesos que permiten a las personas interactuar y compartir en diferentes acontecimientos de la vida, como funerales, rogativas de agradecimiento o ceremonias de fortalecimiento de lazos de amistad. Las principales ceremonias que se mencionan son el gijatun (ceremonia de agradecimiento), el eluwün (funeral), el mafün (casamiento) y el konchotun (visita de amigos/as).

\begin{tabular}{|c|c|c|}
\hline Código & Frecuencia & \% \\
\hline Gijatun & 29 & 40,3 \\
\hline Eluwün & 17 & 23,6 \\
\hline Mafün & 14 & 19,4 \\
\hline Konchotun & 12 & 16,6 \\
\hline Total & 72 & 100 \\
\hline
\end{tabular}

Tabla 2. Subcategoría Ceremonias socioculturales

El gijatun (ceremonia de agradecimiento), se relaciona con agradecer y pedir por la vida, la naturaleza y el trabajo. Se constata que este código presenta una frecuencia de uso del 40,3\%. El gijatun se hace para desarrollar una vida en armonía con el creador, con el medio natural, cultural, social y espiritual. En todo el proceso la referencia es Günechen (fuerza creadora) a quien se le ruega en relación a las actividades cotidianas como son las siembras, para tener buenas cosechas y pedir por la salud de las personas. Un testimonio señala que "antes se hacia un gijatun y Günechen lo escuchaba, ahora no" (K6 [50:50]). Esto podría indicar que en la medida que existe el saber y el conocimiento permite a las personas mantener una relación en armonía con la fuerza creadora, con el medio social y con la naturaleza. Es por esta razón que, por ejemplo en el gijatun, se requiere que las personas participen siguiendo las normas presentes en el saber y conocimiento mapuche. Los niños participan del gijatun (ceremonia de agradecimiento) para observar los diferentes momentos, la ubicación de las personas en el espacio, escuchar el jejipun (rogativa) y los procedimientos implicados. El propósito es que los niños logren comprender la forma en que se desarrolla la ceremonia y retener su sentido cultural y socio religioso. Esto se evidencia en el testimonio de los kimches al señalar que "mi padre siempre se arrodillaba y rogaba en voz alta. Nosotras como estábamos pequeñas lo observábamos y cuando ya hablábamos bien el mapunzugun, comenzamos a realizar nuestras propias rogativas" (K1 
[73:73]). A su vez, implica un comportamiento en coherencia con normas que son básicas y vitales para el gijatun. Por ejemplo, no reírse durante el desarrollo de la ceremonia o "sacarse los zapatos para realizar los procedimientos como realizar el jejipun (rogativa) y el baile" (K14 [36:37]). Esta es una forma de relación de respeto del ser mapuche con la naturaleza y en definitiva con Günechen. Al respecto, se plantea la necesidad que los niños vayan al gijatun para desarrollar algunas disposiciones para el aprendizaje como es escuchar, aprender a observar y memorizar. Un testimonio señala: "también debían ayudar a los padres, pero lo más importante es que ellos fueran socializándose con estas actividades" (K12 [21:22]). Así, las ceremonias socioculturales como el gijatun son instancias que permiten la construcción de conocimiento en la familia mapuche, al vincularse directamente con la aplicación de saberes culturales y espirituales.

En tanto el eluwün (funeral), se refiere a la ceremonia de despedida y sepultura de una persona fallecida. Se constata que este código presenta una frecuencia de uso del 23,6\%. De esta manera, cuando fallece una persona se conversa sobre los principales hitos y saberes asociados a su vida, su relación de parentesco con la familia y la comunidad. El propósito es realizar un análisis retrospectivo sobre la experiencia de vida de la persona fallecida, para traducirlo en una experiencia de aprendizaje y revitalización de la memoria individual y social sobre la historia familiar, comunitaria y sociocultural aportado por ella. En la ceremonia del eluwün: "Las personas que asisten se acuerdan de lo que hizo la persona fallecida, si era un buen hombre o no, una buena mujer o no, según la cultura mapuche..." (K3 [24:24]). La participación de los niños en el eluwün depende del contexto en el que se encuentre la familia. Así, cuando un niño participa del eluwün puede ir “... aprendiendo, cómo se hacía un funeral, a quiénes se atendía, la forma de atender a la familia, reconocer a los parientes que vienen de otros lugares; los dolientes y los amigos" (K14 [41:41]). En consecuencia, en la realización del eluwün las personas se reúnen, comparten y recuerdan la vida del difunto en los distintos ámbitos en que contribuyó al desarrollo de la familia y la comunidad.

El mafün (casamiento) es la ceremonia referida al casamiento desde la lógica del conocimiento mapuche. Se constata que este código presenta una frecuencia de uso del 19,4\%. En la realización del mafün se implican los abuelos, los tíos paternos, maternos y parientes cercanos de la novia y del novio. En la conversación preparatoria del mafün no se implica directamente el padre o la madre del novio con los padres de la novia, salvo excepciones, debido a que hay intereses y susceptibilidades de orden político y económico que lo deben resolver personas que no están comprometidas emocionalmente. Normalmente, la primera conversación la realiza un abuelo, un tío o un pariente cercano que ya tiene experiencia y el saber cultural que le permite realizar una buena negociación. En la conversación se le avisa y se le pregunta al padre de la novia qué animal requiere que le paguen, desatacándose que: "Él decía que debía ser con un caballo, ropa, en aquellos tiempos porque había exigencia. Y toda la familia tenía que ser favorecido con el pago" (K3 [27:27]). Entonces al efectuarse el mafün el padre de la hija acuerda el pago de diferentes especies (animales, mantas, plata) por la entrega de su hija, siendo necesario que se produzca un acuerdo entre las partes y luego se lleva a cabo la ceremonia. Además cuando los jóvenes se casan "...se les aconseja cuando llegaban con esposa. Así, cuando se va a conversar sobre el gijañzugu (el pago), el papá invita a un hombre mayor, alguien que sepa más, un hombre más sabio, entonces se pasarán la mano derecha al recibirlo, debido a que hay que aconsejarles de todo" (K12 [38:38]). En efecto, 
en el desarrollo de la ceremonia las personas observan y participan de consejos hacia la novia y el novio para consolidar el conocimiento sobre el parentesco como una alianza y un espacio para la negociación económica y política, que deberá perdurar en el tiempo.

El konchotun (visita de amigos/as) se reconoce como una ceremonia que fortalece los lazos de amistad entre dos personas. Se constata que este código presenta una frecuencia de uso del 16,6\%. Por medio del konchotun las personas se expresan cariño, visitándose, compartiendo alimentos y entregándose regalos. Al respecto un testimonio señala: “...Mi madre se hizo konchotun con una amiga para no decirse únicamente hermana y para entenderse bien. Eso hacían los antiguos" (K3 [11:12]). El término que se usa para sellar la relación de amistad es koncho, en referencia a la consolidación de una amistad y cariño. Después de un año, desde la fecha en que la persona recibió a su koncho, a la persona le toca devolver lo recibido visitándola igualmente: "De la misma manera lleva lo que recibió, celebran, se hace de todo, mushka (chicha de maíz) también" (K13 [97:97]). Entonces, la visita implica una muestra de afectividad que es recíproca y que permite fortalecer los lazos familiares o de amistad, manteniéndose una comunicación continua en el tiempo donde el regalo simboliza la expresión de cariño entre las personas.

En consecuencia, las ceremonias socioculturales son instancias que permiten la construcción de conocimiento en la familia mapuche, al vincularse elementos sociales, culturales y espirituales en la realización de este tipo de actividades.

\subsubsection{Subcategoría Objetos de conocimiento}

La subcategoría considera los objetos que permiten construir conocimiento durante la educación familiar de los niños. Estos objetos de conocimiento son tangibles e intangibles y se relacionan con aspectos sociales, culturales y naturales. Es así que los kimches señalan como objetos de conocimiento el medio natural, las fuerzas espirituales, Günechen (fuerza creadora) y los pewma (sueños).

\begin{tabular}{|c|c|c|}
\hline Código & Frecuencia & \% \\
\hline Medio natural & 32 & 48,5 \\
\hline $\begin{array}{c}\text { Fuerzas } \\
\text { espirituales }\end{array}$ & 15 & 22,7 \\
\hline Günechen & 11 & 16,7 \\
\hline Pewma & 8 & 12,1 \\
\hline Total & 66 & 100 \\
\hline
\end{tabular}

Tabla 3. Subcategoría Objetos de conocimiento

El conocimiento sobre el medio natural se relaciona con la observación y relación directa con el contexto territorial en el que se desarrolla la vida de la familia mapuche. Se constata que este código presenta una frecuencia de uso del 48,5\%. Las personas construyen conocimientos que utilizan en su diario vivir. Para el mapuche, las plantas, animales, cerros y vertientes, son fuentes de conocimiento que definen formas de interacción de las personas con el medio en el que se desenvuelven. En este proceso, la relación de las personas con cada elemento de la naturaleza, cada cosa que se ve en la 
naturaleza, tiene un significado para su vida. Por ejemplo, el territorio, "puede permitir conocer el tuwün, el küpan de las personas y cómo se puede avanzar más allá..." (K19 [7:7]). En este sentido, las personas obtienen de la naturaleza información necesaria para realizar sus actividades cotidianas. Al mismo tiempo, una interpretación adecuada del comportamiento de sus elementos naturales o la biodiversidad permite comprender lo que podría ocurrirle a las personas en la relación con el medio. De esta forma, un testimonio señala que: “...Yo viví una experiencia con el pekeñ (búho) cuando estaba cuidando a mi mamá. Llegó un pekeñ y se sentó aquí cerca y a los dos o tres días falleció mi mamá..." (K2 [88:89]). Entonces las personas interpretan los mensajes positivos y negativos que entrega la naturaleza, estableciéndose una relación de complementariedad. Esta interpretación varía según los significados que se le atribuya a los anuncios, de acuerdo al contexto en el que se encuentre la persona y al saber cultural que posea. Por ejemplo un testimonio señala: “...ese maqui sirve para teñir lana. Entonces hay cosas de la naturaleza que sirven mucho, pero a veces cuando la gente no sabe, anda por encima de las cosas y por no haber aprendido, por no haber sabido, no usa esos materiales" (K17 [22:22]). Aquí, se remarca la visión integrada del mapuche con respecto a que en la naturaleza todo sirve para algo.

El código fuerzas espirituales establece que en la familia mapuche a través de aquellos seres que no son visibles físicamente, es posible construir conocimientos. Se constata que este código presenta una frecuencia de uso del 22,7\%. De esta forma, los seres protectores de los espacios naturales como el gen majiñ (área cubierta por agua con menos de un metro de profundidad, con circulación permanente), gen wigkul (dueño del cerro), gen wixunko (dueño de las vertientes) y gen menoko (área cubierta por agua que tiene una profundidad de hasta unos 6 metros, normalmente no tiene circulación superficial, aunque tiene circulación subterránea), permiten a los sujetos orientar y prevenir riesgos naturales en la interrelación de las personas con los elementos espirituales del medio natural. Mientras mayor sea el conocimiento de las personas sobre el saber, normas culturales y espirituales que definen los aspectos esenciales de las fuerzas espirituales que sustentan el orden y la relación de armonía con la naturaleza, más riesgos es posible prevenir. Estos saberes se relacionan con los diferentes tipos de seres que se identifican en el medio natural y los mensajes que portan, lo cual definen formas de interactuar en el contexto. Un testimonio señala que: “...Mi mamá cada vez que iba a lavar en el canal, decía que hay una cosa que le dicen gakiñ (tipo de anfibio), canta parecido a una rana. No hay que molestarlo, porque te podría castigar. Es el dueño del agua, nunca hay que molestarlo, principalmente cuando se trata de una mujer embara$z a$ " (K14 [118:119]). Entonces, las fuerzas espirituales se asocian con la generación de enfermedades o castigos cuando las personas no respetan su presencia en un determinado espacio. El conocimiento que subyace es que hay determinados contextos naturales como vertientes de agua, humedales y pendientes pronunciadas, que podrían poner en riesgo la circulación de una mujer embarazada, al ingresar a dichos espacios naturales. Por lo cual es de vital importancia crear conciencia en los niños sobre estos conocimientos, para que puedan desenvolverse en forma adecuada en el medio natural.

El código Günechen (fuerza creadora) se considera como otro referente de conocimiento en la familia, al obtener de él los conocimientos que se necesitan en la vida diaria. Se constata que este código presenta una frecuencia de uso del 16,7\%. De esta forma, los kimches plantean que los mapuches realizan muy bien sus rogativas a Günechen 
y es por eso que los escucha, porque además hay respeto "se respeta las normas, los saberes, los alimentos, se saluda bien la gente" (K1 [43:43]). De ahí es que exista la necesidad de comunicarse con Günechen, para no perder su guía, ya sea al viajar, trabajar la tierra, al ingresar a los espacios naturales. Esta comunicación se realiza a través de una oración, "porque sin él no podríamos vivir, debemos encomendarnos a nuestro Günechen. Incluso, cada vez que uno sale de la casa hay que encomendarse, para que nos vaya bien en nuestras diligencias" (K3 [57:57]). Es por medio de Günechen que se establece el equilibrio en la vida de las personas, una relación de equilibrio con la naturaleza, con las fuerzas espirituales, como fuente de conocimiento para el desarrollo de la familia y la comunidad.

El código pewma (sueños) de las personas permiten la construcción de conocimiento a través de la representación de hechos o situaciones que todavía no ocurren, como también la comunicación espiritual con familiares fallecidos. Se constata que este código presenta una frecuencia de uso del 12,1\%. Es así que los kimches indican que los sueños son una actividad mental e intelectual previsora sobre el devenir de las personas, el mejoramiento de prácticas y de actuación adecuada de los sujetos en los procesos de relación social y el desarrollo de respeto con los elementos del medio. Es una forma de aprendizaje y de enseñanza que implica a las personas en su dimensión subjetiva e intersubjetiva para descubrir el futuro como individuo y como colectividad con respecto a lo que podría pasar y suceder en su contexto específico y global. En las personas se valora el hecho de soñarse como una posibilidad de seguir aprendiendo. Un testimonio señala que: "Yo escuchaba, que mi abuelita me decía de esa forma tienes que hacer la rogativa, en todos lugares que vayas. Eso mismo, uno lo aprende mejor a través del sueño, porque ahí a uno le enseñan mejor" (K9 [19:19]). El obtener conocimiento de los sueños implica que las personas sepan interpretar los avisos que se le presentan, ya que así se logra responder a necesidades que se presentan en la vida diaria.

\section{DISCUSIÓN}

Las diferencias observadas en las lógicas de construcción de conocimiento en los contenidos y finalidades educativas se relacionan con el pensamiento que subyace en el proceso de formación de la persona mapuche y no mapuche. Según los kimches, la formación de la persona mapuche buscar dotar a los niños y jóvenes de saberes y conocimientos que les permitan relacionarse en armonía con su entorno natural, social, cultural y espiritual. La naturaleza de los contenidos busca formar personas que respondan al referente kimche, cuyas cualidades se relacionan con el dominio de saberes, conocimientos y actitudes que dan cuenta de una correcta formación familiar. Los objetivos son lograr el dominio de saberes y conocimientos para que los sujetos sean respetados en la familia y en la comunidad. También se busca mantener una buena imagen personal y familiar a través de actitudes y acciones correctas de personas vinculadas por parentesco. Además, mantener y dar continuidad a las ceremonias y prácticas sociales y culturales del patrimonio cultural mapuche.

En este contexto, la diferencia entre una forma y la otra radica en las razones que tienen ambas bases sociales para concebir los aprendizajes, la construcción del conocimiento y la lógica para formar y educar a niños y jóvenes. La educación occidental es 
de orden abstracto cuyo objetivo es formar a las personas para el trabajo (producción económica) y la educación mapuche es más trascendental y prepara para relacionarse en armonía con el medio natural, social, cultural y espiritual. En la familia mapuche los encargados de la formación de personas buscan mantener una imagen personal y familiar. En este proceso, las acciones y actitudes asumidas por las generaciones jóvenes de las familias dan cuenta de la forma en que se llevó a cabo su educación. Por lo tanto, los responsables serán dignos de respeto y valoración social. Desde la educación escolar, según las observaciones etnográficas, si bien los profesores le otorgan énfasis al proceso de formación de los niños, al ser éste un proyecto amplio no se involucran como personas. Entonces los docentes en la actualidad solo se limitan a 'pasar' contenidos a su manera, en base a conocimientos occidentales que en la escuela se asumen definidos. En cambio, desde la educación familiar mapuche los conocimientos se construyen en forma permanente, según contexto y experiencia de relación directa con el medio, fundamentado en la memoria individual y social.

Existe otro aspecto que da cuenta de una diferencia de racionalidad y que incide directamente en la construcción de conocimientos en ambos grupos sociales, mapuches y no mapuches. En la familia y en la comunidad, los niños participan de manera progresiva en actividades, asumen responsabilidades en la medida que van adquiriendo nuevos conocimientos, lo cual va en directa relación con la edad. De acuerdo con la información, a la edad de 14 años en promedio, los jóvenes son dignos de respeto y están en condiciones de asumir responsabilidades mayores, las cuales se fortalecen al momento de contraer matrimonio. En la sociedad occidental, sin embargo, este proceso se realiza a través de una formación que pretende ser lúdica y los aprendizajes sólo se 'usan' como el requisito para pasar a otro nivel de enseñanza. Una vez que los jóvenes tienen 18 años son considerados dignos de desarrollarse en forma más autónoma. Este proceso, sin embargo, se realiza en un contexto abstracto de aprendizajes, a diferencia de la familia, en donde se generan aprendizajes en contextos reales y los niños son considerados desde pequeños, por ejemplo, en el saludo. Tanto a los adultos como a los niños se les debe saludar según las distinciones de género y se debe manifestar el respeto en la interacción con personas mayores. Así, los jóvenes se asumen como personas en la medida en que se incorporan a las prácticas sociales con los adultos y kimches. En ese sentido, la educación intercultural debiera dar cuenta de las lógicas de construcción de conocimiento y racionalidades del pensamiento mapuche para diseñar y ejecutar procesos de enseñanza y aprendizaje con pertinencia cultural. La tendencia que se constata es una desvalorización de saberes y conocimientos propios en las familias mapuches por parte de las nuevas generaciones. Sin embargo, los datos indican que los kimches consideran importante que las personas mapuches cuenten su historia en la escuela. Entonces es posible inducir la necesidad de generar una relación entre la familia, la comunidad y la escuela, para identificar conocimientos deseables de incorporar a la educación escolar, como una participación en niveles de decisión de un posible currículum escolar intercultural.

Si bien los conocimientos están en la memoria de los sabios de las familias y comunidades, es necesario que las nuevas generaciones aprendan y comprendan aspectos fundamentales. Por ejemplo, incorporar el respeto según la cosmovisión mapuche como contenido en la educación escolar, permitirá desarrollar una actitud positiva hacia el aprendizaje intercultural en los alumnos mapuches y no mapuches. En este proceso, se constata el conocimiento del mapunzugun (lengua mapuche), como un medio posible 
para el aprendizaje y la enseñanza de la racionalidad propia a través de la explicitación del sentido que tiene un contenido educativo mapuche. Mientras que el pentukun (saludo protocolar) es lo que le permitirá a los niños tener un correcto diálogo con las personas en la comunidad. Para lograr estos aprendizajes es necesario generar instancias en las cuales los niños puedan hacer uso de estos contenidos, ya sea en la escuela o en la comunidad. Es decir, llevar la escuela a la comunidad y viceversa, donde los niños tengan la oportunidad de participar de actividades socioculturales mediadas por el educador/a tradicional, que denominamos kimche, lo cual permita enriquecer la formación intercultural de personas mapuches y no mapuches.

En la familia, el kimche es la figura encargada de la formación de niños y jóvenes, puesto que posee conocimientos y características personales para guiar la construcción de conocimientos. Mientras que los profesores actuales, en general, no tienen conocimientos mapuches para incorporarlos al proceso de enseñanza y aprendizaje escolar. En este contexto, en las familias se refleja la imperiosa necesidad de que la incorporación de conocimientos mapuches a la escuela sea a través del educador/a tradicional para abordar dichos conocimientos con propiedad. Así también, se constata que ésta persona no sólo debe tener conocimiento, sino que debe practicarlo, ya sea usando el mapunzugun en sus conversaciones cotidianas en la comunidad, visitando a los familiares y participando en actividades socioculturales que allí ocurran. Los saberes y conocimientos mapuches que debería dominar un kimche no sólo debiera transmitirlo a las nuevas generaciones, sino también demostrar, con sus acciones y actitudes, ser la persona idónea para asumir un rol de educador. Además, la figura del educador/a tradicional será el referente que los niños tendrán para observar, aprender y desenvolverse en competencias escolares interculturales.

Todo este proceso se debiera realizar basado en una lógica que responde a la racionalidad propia del pensamiento mapuche. El mapunzugun es uno de los elementos que caracteriza esta lógica, dado que a través de este se transmite el sentido del patrimonio social, espiritual y cultural mapuche. De esta manera, la integración de saberes y conocimientos mapuches a la enseñanza escolar incorporaría no sólo contenidos educativos, sino también una lógica de construcción de conocimiento y racionalidad propia. De acuerdo con las observaciones etnográficas y entrevistas a kimches, el conocimiento mapuche que se debiera considerar en la escuela se relaciona con tres ámbitos: el relacionado con la naturaleza, con la sociedad y con las fuerzas espirituales. En este sentido, se pone de manifiesto el desconocimiento por parte de niños y jóvenes de contenidos educativos que tienen relación con estos ámbitos. En relación con el conocimiento sobre las plantas, se devela que en la actualidad se desconoce tanto las propiedades como el uso de las plantas. Aunque un porcentaje significativo señala que estos contenidos educativos están presentes en la escuela. Todo indica que se trataría de la incorporación del entorno natural más próximo, pero no desde la lógica mapuche. Por lo tanto, no se estarían abordando temas relacionados con la naturaleza, las personas y las fuerzas espirituales. Además, se devela que los niños hoy día desconocen contenidos para relacionarse con la familia y la comunidad, que se manifiesta por la ausencia de contenidos tales como el respeto, el pentukun (saludo protocolar) y las relaciones de parentesco. Esta situación se debería, principalmente, a que la escuela no valora contenidos educativos mapuches, debido a que se espera que los alumnos aprendan conocimientos que son valorados para desenvolverse en la sociedad nacional. 
Entonces, las diferencias se relacionarían con las finalidades, los fundamentos, los contenidos educativos y su proceso de construcción. Por una parte, la educación escolar busca que los niños y jóvenes se apropien de herramientas para desenvolverse en la sociedad nacional. Por otra parte, las nuevas generaciones mapuches han desestimado los contenidos educativos fundamentales como el respeto y el conocimiento sobre el mapunzugun. Este problema es producto de una escolarización que cada día ocupa más tiempo y espacio en la formación de personas, sin considerar saberes y conocimientos propios (mapuche y no mapuche) como contenidos valóricos para generar procesos de relaciones interculturales.

\section{CONCLUSIONES}

Un currículum intercultural en contexto indígena se basa en contenidos educativos que consideran las lógicas de construcción del conocimiento vernáculo y aquellos propios del marco curricular. De esta forma se toma en cuenta la manera de concebir el conocimiento que fundamenta la formación de personas que podrán vivir y relacionarse interculturalmente con sujetos y grupos que poseen otra cultura. Así, la enseñanza y el aprendizaje de dichos conocimientos en el medio escolar permitiría a las nuevas generaciones de mapuches y no mapuches desenvolverse en contextos interculturales, en base a un conjunto de habilidades cognitivas y valóricas posibles de desarrollar en el plano individual y social. En efecto, el currículum intercultural tiene como finalidad develar, organizar, sistematizar y planificar contenidos educativos propios de los educandos, para generar la relación de saberes en los procesos de enseñanza y aprendizaje a partir de la educación familiar y la educación escolar.

Entre los pueblos indígenas, en general, el saber y el conocimiento están relacionados directamente con el mundo natural, con las redes de parentesco en la comunidad y con los conocimientos sobre el universo. Estos saberes se van construyendo junto con el desarrollo histórico de los pueblos y son producto de un proceso de creación mental y cognitiva que configuran progresivamente el patrimonio cultural objetivo y subjetivo. De esta manera, el patrimonio cultural mapuche, está constituido por elementos tales como: valores, creencias, normas, reglas, saberes y conocimientos propios de tipo objetivo y subjetivo. Estos saberes se asumen como el fundamento de los contenidos educativos para la enseñanza y el aprendizaje intercultural de niños mapuches y no mapuches.

De esa manera, se constata una distancia epistemológica entre la racionalidad mapuche y la occidental, tanto en los modos de construcción del conocimiento, como en el pensamiento para comprender el mundo. Estas racionalidades están en la base del proceso de formación de personas, sea en el ámbito familiar o escolar. La escuela está en el centro de la distancia epistemológica, donde la formación de personas es el nexo de una relación educativa hipotética de ambas racionalidades, históricamente en oposición. Superar esta distancia es el objetivo de la educación y del currículum intercultural.

Entonces, en contexto mapuche, el profesor no puede contar únicamente con los saberes formalizados por la escuela, ya que las prácticas pedagógicas son mucho más complejas y están fuertemente influenciadas por la contingencia de la cultura cotidiana. Además, su propósito no es sólo el conocimiento, sino también las prácticas e ideologías que subyacen en ambas lógicas de construcción de conocimientos educativos, como producto de una deliberación práctica. En esta lógica, la situación pedagógica en contexto intercultural 
es concebida de forma multidimensional e impredecible, ligada a una realidad compleja de la racionalidad que en cierto modo define y fundamenta la formación de personas.

A partir de los resultados es posible proponer un marco general para la educación intercultural, la que debiera asumir los siguientes supuestos: 1) En relación con el proceso de enseñanza-aprendizaje, asumir que las personas adquieren habilidades y destrezas que les permiten vivir en una determinada comunidad, en la cual están inmersos; 2) Las destrezas y habilidades se relacionan con sistemas de significados que capacitan a las personas para comunicarse y con actividades prácticas que se orientan hacia la transformación del mundo natural, social y cultural; 3) Quienes participan de dichas situaciones lo hacen desde sus propias perspectivas y metas que son iguales, pero diferentes en el sentido y significado atribuido a las cosas; 4) Las personas aprenden inmersas en un mundo social, aunque el acto de aprender se considere como un hecho predominantemente individual; 5) Las personas aprenden en las diferencias, lo cual constituye una riqueza de la producción humana que mejora la calidad de los aprendizajes, las oportunidades y las relaciones sociales; 6) Las prácticas pedagógicas interculturales tienen por objeto generar una relación dialógica entre los saberes populares, los saberes indígenas y los saberes y conocimientos escolares; y 7) Debiera centrarse en explicitar aquellos aspectos que son diferenciadores de ambas culturas en sus diferentes dimensiones y revalorizar las particularidades de cada una para generar una relación intercultural fundada en la deliberación.

\section{REFERENCIAS BIBLIOGRÁFICAS}

Ardoino, J. (2005). Complejidad y Formación. Pensar la educación desde una mirada epistemológica. Buenos Aires: Novedades Educativas.

Bisquerra, R. (2000). Métodos de investigación educativa. Guía práctica. Barcelona: CEAC.

Bisquerra, R. (2004). Metodología de la investigación educativa. Madrid: La Muralla.

De Camilloni, A. (2001). Los obstáculos epistemológicos en la enseñanza. Barcelona: Gedisa.

Gauthier, C., Desbiens, J., Malo, A., Martineau, S. y Simard, D. (1997). Pour un Théorie de la Pédagogie. Recherches Contemporaines sur le savoir de enseingants. Québec: Le Presses de 1'Université Laval.

Guerra, M. (2006). Los científicos y su trabajo en el pensamiento de los maestros de primaria. Una aproximación pedagógicamente situada. Revista Mexicana de Investigación Educativa, vol. XI, n. 31, 1287-1306.

Hessen, J. (2003). Teoría del conocimiento. México: Editorial Tomo.

Izquierdo, A. (1999). Espacio-temporalidad y omnijetividad. Una aproximación Epistemológica. Revista Nómadas, n. 11, 241-247.

Legendre, R. (1993). Dictionnaire actuel de l'éducation. Montréal: Guérin Éditeur limitée.

Krippendorff, K. (1990). Metodología de análisis de contenido. Teoría y práctica. Barcelona: Paidós. Nadeau, R. (2009). Philosophies de la connaissance. Montreal: Les Presses de l'Université Laval. Pérez, G. (2000). Investigación cualitativa. Retos e interrogantes. Madrid: Colección Aulas Abiertas. Quilaqueo, D., Fernández, C. y S. Quintriqueo (2010). Interculturalidad en contexto mapuche. Neuquén: Educo.

Quintriqueo, S. (2002). Representación de la relación de parentesco que la familia mapuche posee y su transmisión a niños mapuches de edad escolar primaria en relación con los contenidos de Formación Inicial Docente. Tesis de Maestría en Educación realizado, À l'Université du Québec en Abitibi-Temiscamingue, Québec. 
Quintriqueo, S. y Cárdenas, P. (2010). Educación Intercultural en contextos mapuches. Articulación entre el conocimiento mapuche y el occidental en el ámbito de las ciencias. En D. Quilaqueo, C. Fernández y S. Quintriqueo (eds.), Interculturalidad en contexto mapuche (pp. 91-128). Neuquén: Educo.

Quintriqueo, S. (2010). Implicancias de un modelo curricular monocultural en contexto mapuche. Santiago de Chile: LOM Editores.

Quivy, R. y Campenhoudt, L. (1998). Manual de Investigación en Ciencias Sociales. México: Limusa.

Rodrigo, M. J. y Arnay, J. (1997). La construcción del conocimiento escolar. Barcelona: Ediciones Paidós.

Rodríguez, G., Gil J. y García E. (1999). Metodología de la investigación cualitativa. Málaga: Ediciones Aljibe S.A.

Restrepo, E. (2004). Esencialismo étnico y movilización política: tensiones en las relaciones entre saber y poder. En O. Barbary y F. Urrea (eds.), Gente negra en Colombia. Dinámicas sociopolíticas en Cali y el Pacífico (pp. 227-244). Medellín: Centro de Investigaciones y Documentación Socioeconómicas de la Universidad del Valle.

Sagastizábal, Ma. (2006). Comprender la escuela hoy. Sociedades multiculturales y realidades educativas complejas. En Ma. Sagastizábal, C. Perlo, B. Pivetta y P. San Martín (eds.), Aprender y enseñar en contextos complejos: multiculturalidad, diversidad, fragmentación (pp. 11-41). Buenos Aires: Noveduc.

Schmelkes, S. (2006). El conocimiento campesino. Revista Mexicana de Investigación Educativa, vol. XI, n. 31, 333-337

Torres, H. y Quilaqueo, D. (2010). Concepción de tiempo en el discurso educativo de los kimches. En D. Quilaqueo, C. Fernández y S. Quintriqueo (eds.), Interculturalidad en contexto mapuche (pp. 257-278). Neuquén: Educo. 